\title{
Photonic crystal waveguides by direct writing of e-beam on self-assembled photonic crystals
}

\author{
SUNITA KEDIA and R VIJAYA* \\ Department of Physics, Indian Institute of Technology Bombay, Mumbai 400 076, India
}

MS received 21 May 2008; revised 31 January 2011

\begin{abstract}
Direct electron beam lithography technique is used for writing a variety of waveguide structures on thin films of polymethyl methacrylate (PMMA) and self-assembled three-dimensionally ordered photonic crystals made up of PMMA colloidal spheres. The waveguide structures fabricated on both these type of samples are characterized by scanning electron microscope and optical microscope images.
\end{abstract}

Keywords. Photonic crystals; waveguides; electron beam lithography.

\section{Introduction}

Photonic crystals consist of a periodic arrangement of dielectric materials. When an electromagnetic wave of a wavelength comparable to the periodicity of the crystal is incident on a photonic crystal, it undergoes multiple scattering. Depending upon the parameters such as the index contrast and the periodicity of a given crystal, the scattered wave travelling in the appropriate direction undergoes constructive interference. The crystal thus shows high reflection and a corresponding low transmission for a particular range of wavelength. This wavelength range is called the stop band (or the pseudo-gap) for that photonic crystal and the crystal becomes a photonic bandgap material if the stop band overlaps for different angles of incidence for both the TE and TM polarizations (corresponding to a complete bandgap) (Yablonovitch 1987; John 1987; Joannopoulos et al 1995). These structures show a stop band in the visible or near-IR wavelength region if they are designed with a sub-micron periodicity in the dielectric constant. They have several applications in optics and photonics such as in designing low threshold lasers, high efficiency micro lasers (John and Quang 1995) and light emitting diodes (Yablonovitch 1993).

The diversity of applications can be increased if an intentional point defect or a line defect or a planar defect can be created in the photonic crystals. There are different ways of introducing defects into the structure, such as by doping the crystal with impurities or by the addition or removal of high index dielectric materials by different processes, which can change the optical property of the crystal in a controlled manner. Such designs are especially required for low mode volume resonators with a high quality factor in nanophotonic applications. Photonic crystal technology is

\footnotetext{
*Author for correspondence (rvijaya@phy.iitb.ac.in)
}

capable of providing compact designs of integrated optical devices if micron-scale waveguides can be efficiently fabricated in them. Such waveguide structures designed on threedimensionally ordered photonic crystals provide the confinement and guidance of electromagnetic waves in the range of the stop band of the crystal if an allowed guided mode exists. The waveguide structure can be fabricated by different techniques such as electron beam lithography (EBL). EBL has been used by different groups for the fabrication of two-dimensional photonic crystals and photonic crystals with waveguide structure (Panepucci et al 2004; Stodolka et al 2005). Very few reports are available where the EBL is used for patterning three-dimensional colloidal photonic crystals (Ferrand et al 2004).

In the present work, the waveguide structures are patterned on polymeric thin film and three-dimensionally ordered photonic crystal made from colloidal spheres of PMMA, using the EBL technique. When a channel waveguide structure is written on a PMMA photonic crystal, the light is guided through the rarer medium (air channel) due to the bandgap property of the photonic crystal surrounding it, as opposed to the case of a conventional waveguide where the light guidance is possible by total internal reflection in the high index core surrounded by a relatively lower-index cladding.

\section{Experimental}

\subsection{Materials}

PMMA of molecular weight $495 \mathrm{~K}$ is dissolved in $8 \%$ anisole (A) (Microchem) and used to fabricate thin films on glass substrate. Colloidal suspension of PMMA spheres of diameter $287 \mathrm{~nm}$ (obtained from M/s Microparticles $\mathrm{GmbH}$ ) is used for fabricating the photonic crystals by inwardgrowing self-assembly. 


\subsection{Process}

EBL is a direct writing technique and it provides high resolution and versatile patterns. In this method, a small spot of electron beam writes patterns directly onto a substrate coated with an electron-beam resist, eliminating the expensive and time-consuming production of mask (Tseng et al 2003). The e-beam resists are high molecular weight polymers dissolved in a liquid solvent. The polymer changes its property when exposed to electron beam and gives patterns depending upon the type of resist. The electron-beam resist can be positive (e.g. PMMA) or negative (e.g. SU-8). After exposure to the electron beam, the positive resist will dissolve in the developing solution, and a positive tone pattern is formed on the substrate. On the other hand, the negative resist becomes insoluble in the developing solution after exposure, while the unexposed part will be dissolved.

Before writing the waveguide structures on photonic crystals made of PMMA colloids, the parameters of EBL experiment were optimized on PMMA thin film due to nonavailability of these quantities from earlier works of others. Different waveguide structures such as straight strip waveguide, strip waveguide with a bend and distributed Bragg reflector (DBR) (Mukherjee et al 1995) structure have been written on thin films for different accelerating voltages and beam currents. The fabricated patterns are structurally characterized by scanning electron microscope (SEM) and optical microscope $(\mathrm{OM})$ images. Three-dimensional photonic crystals are grown by the method of self-assembly and characterized by both structural and optical methods. The single channel waveguide structure is written on the photonic crystal with the same EBL system by changing the parameters slightly around the earlier optimized conditions.

\subsection{EBL on PMMA thin film}

An appropriate volume of the resist solution is dropped on the substrate and spun at a high value of rotations per min to get the thin film. The sample is pre-baked at a high temperature in the oven to evaporate the casting solvent. Final thickness of the film is measured by profilometer. In the present work, PMMA with a molecular weight of $495 \mathrm{~K}$ with $8 \%$-A is spin-coated on clean glass substrates at 1000 rotations per min to get uniform films of $1 \mu \mathrm{m}$ thickness. Samples are heated at $160^{\circ} \mathrm{C}$ for $30 \mathrm{~min}$ in an oven and subsequently sputtered with a thin film of gold for eliminating the charging effect during EBL. Exposure of patterns on the film was performed with the Raith ELPHY Plus JSM-6400 EBL system with accelerating voltages ranging from $20 \mathrm{kV}$ to $30 \mathrm{kV}$ and with beam currents from $100 \mathrm{pA}$ to $250 \mathrm{pA}$. The accelerating voltage controls the penetration depth, and the beam current controls the writing area.

The exposed samples were developed in a 1:1 mixture of methyl-isobutyl-ketone (MIBK) and isopropanol (IPA) for $30 \mathrm{~s}$ and then rinsed in IPA for $30 \mathrm{~s}$ to stop the development process. PMMA is a positive resist and it becomes more soluble in the developing solution after being exposed to the electron beam (Tseng et al 2003). During the developing process, the developer solvent washes away the exposed portion of the structure thus forming an air channel. Since conventional light guidance requires higher index waveguides, two such air channels are written parallel to each other, with the intermediate region serving as the waveguide. Finally the samples were dried in a flow of nitrogen. A variety of waveguides are written on different samples by this method.

\subsection{EBL on PMMA photonic crystal}

Three-dimensionally ordered photonic crystals are grown within $3 \mathrm{~h}$ using the colloidal solution of PMMA (colloidal sphere diameter $287 \mathrm{~nm}$ ) by the inward growing selfassembly method (Yan et al 2005). This method provides face centred cubic close-packed arrangement of the colloidal globules. A post-fabrication thermal treatment provides mechanical strength to the crystals. Details of the fabrication procedure are given elsewhere (Nair and Vijaya 2008). An area of $3 \times 3 \mathrm{~mm}^{2}$ with a thickness of about $2 \mu \mathrm{m}$ of the crystal is exposed to the electron beam. Different accelerating voltages ranging from $2 \mathrm{kV}$ to $20 \mathrm{kV}$ with a beam current of $70 \mathrm{pA}$ to $100 \mathrm{pA}$ and dose of $200 \mu \mathrm{m} / \mathrm{cm}^{2}$ were tried for the experiment (Ferrand et al 2003). The optimized conditions for our samples were found to be an accelerating voltage of $5 \mathrm{kV}$ at a beam current of $80 \mathrm{pA}$ with a dose of $200 \mu \mathrm{m} / \mathrm{cm}^{2}$. After exposure, the photonic crystal samples are developed in IPA solution for $30 \mathrm{~s}$ and dried in nitrogen flow. The exposed portion is removed leaving an air channel. The channel is written in a controlled way so that the electron-beam modifies a selected thickness of the sample. An air channel of rectangular cross-section is obtained on the crystal which is surrounded by the photonic crystal on three sides. The developing process after the electron beam exposure has been optimized through several repeated trials so that the unexposed portion of the colloidal crystal remains undisturbed. Unlike the PMMA thin film, light guidance is possible in this air channel due to the bandgap effect of the photonic crystal under suitable conditions.

\section{Results and discussion}

The waveguide structures on the photonic crystals and on the PMMA thin film are characterized by SEM and OM images. The quality of the photonic crystal is studied by doing structural and optical characterization of the crystal.

\subsection{Characterization of waveguides on thin film}

Waveguide structures with different dimensions are written on PMMA thin film by EBL technique. The parameters of 


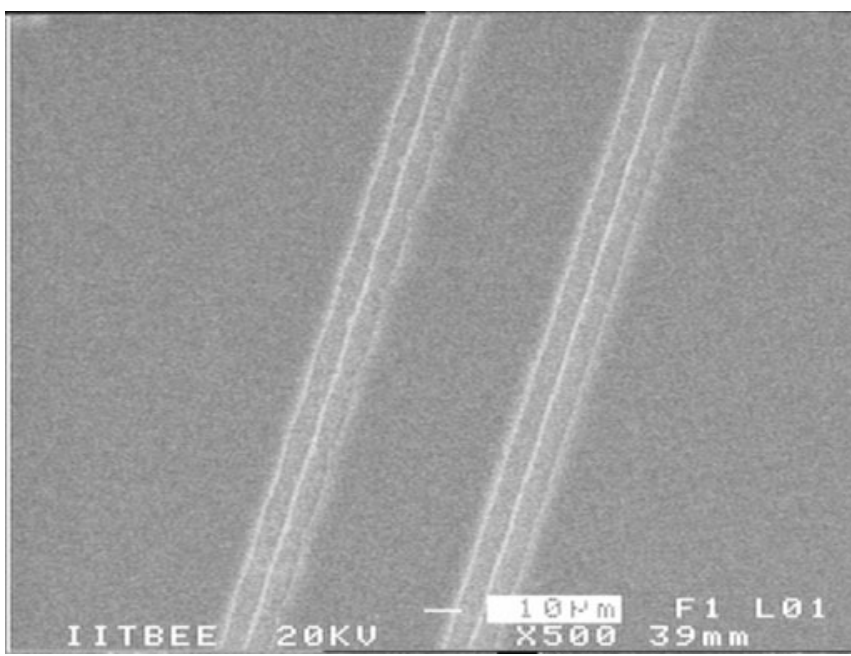

Figure 1. Dielectric strip waveguide on PMMA thin film (scale bar $10 \mu \mathrm{m})$.

the experiment were optimized as per the thickness of the film and the required size of the air channels. Figure 1 is the SEM image of straight dielectric strip waveguide where two air channels of the same size are written parallel to each other (white colour) and the intermediate region (dark colour) is the denser medium through which light can be guided by total internal reflection from the interfaces. The length of the PMMA waveguide is about $3200 \mu \mathrm{m}$ and its width is about $35 \mu \mathrm{m}$. The width of the air channel is about $15 \mu \mathrm{m}$. The structure will behave as a symmetric waveguide (surrounded with air channel at the two sides) for light incident in the plane of the thin film and it will behave as an asymmetric waveguide (surrounded by substrate and air) when light is incident in the plane perpendicular to the plane of the film. Since the refractive index contrast is large between the core and the cladding of the waveguide, it is multimoded at visible and near-IR wavelengths.

Figure 2 is the SEM image of the strip waveguide with a bend of $120^{\circ}$. This bend is introduced to control the number of propagation modes through the waveguide. The critical angle for this waveguide is nearly equal to $42^{\circ}$ and the numerical aperture is 1.1 . Hence, when the light is incident within this numerical aperture, it will be guided through the straight part of the channel. But at the bend, the angle of incidence should be equal to or greater than $60^{\circ}$ for guidance and only those modes incident at an angle greater than $60^{\circ}$ will be guided and other modes will be radiated out. This is an efficient design to reduce the number of guided modes.

In a similar way, a distributed Bragg reflector (DBR) structure is also written on PMMA thin film. A conventional DBR structure contains multiple layers of alternating dielectric materials with two different refractive indices. When the light is incident in the low-index layer, it gets guided due to the periodic variation in the refractive index on either side of that layer leading to constructive interference and not due

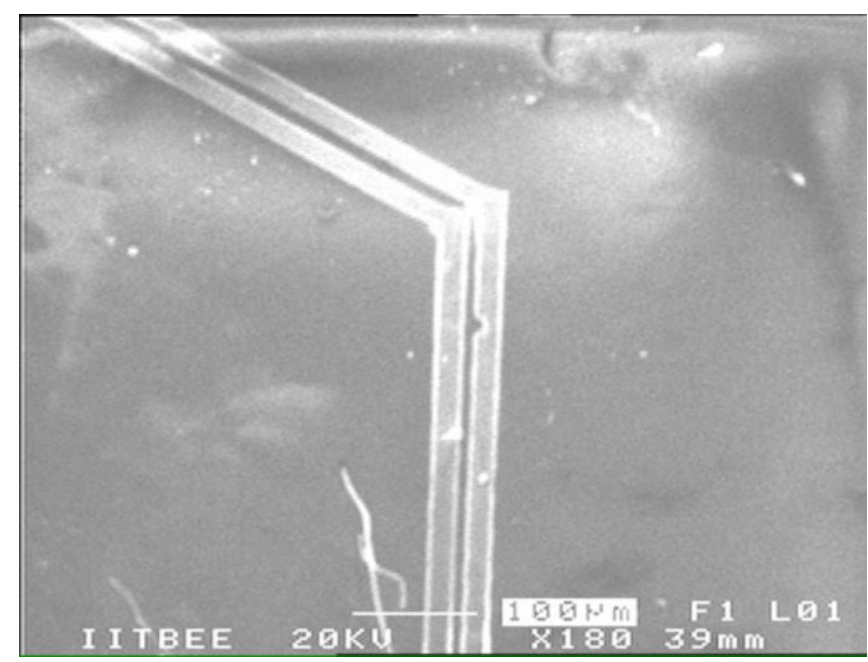

Figure 2. Dielectric strip waveguide on PMMA thin film with $120^{\circ}$ bend (scale bar $100 \mu \mathrm{m}$ ).

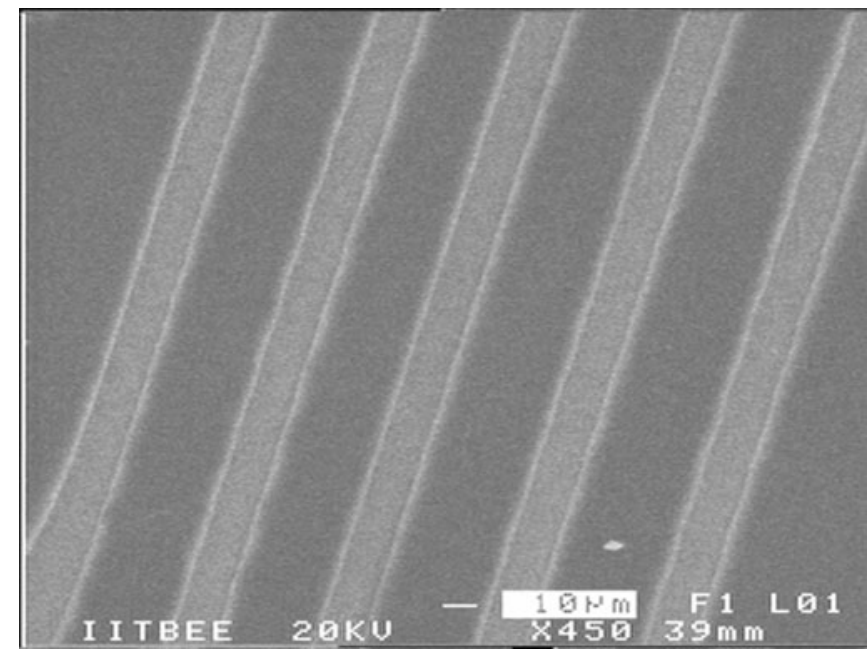

Figure 3. Distributed Bragg reflector on PMMA thin film (scale bar $10 \mu \mathrm{m})$.

to the total internal reflection. In the present work, a number of air channels are written on thin film of PMMA which are equally spaced within the PMMA film. Figure 3 is a SEM image of DBR structure where PMMA strips (black colour) of width about $20 \mu \mathrm{m}$ are separated by air channels (grey colour) of width about $10 \mu \mathrm{m}$. The periodicity is in the plane of the film. If light is launched into the air channel, part of it will be reflected and part of it transmitted at every reflection inside the waveguide, but there will be multiple reflections occurring at the subsequent waveguides on either side. Hence, guidance is possible in a low index waveguide due to the in-plane one-dimensional periodicity. When an electromagnetic wave of wavelength close to four times the optical thickness of the layers is incident into any one channel, the boundary of each layer causes a partial reflection of the wave 


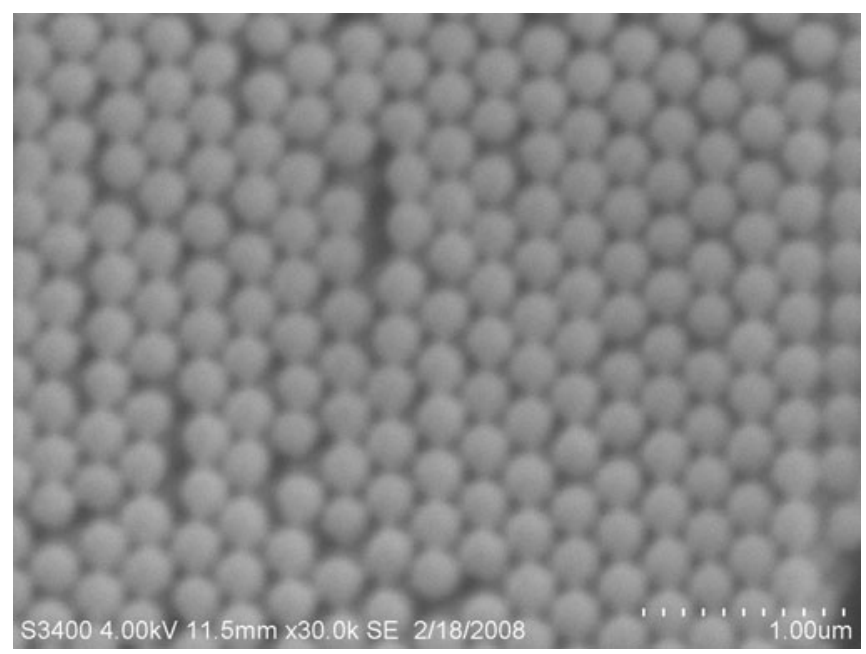

Figure 4. Magnified SEM image of 3D ordered photonic crystal made using PMMA colloidal spheres of diameter $287 \mathrm{~nm}$ (scale bar $1 \mu \mathrm{m})$.

(Fu et al 2003). Many reflections can interfere constructively and the structure can act as a high quality guidance device.

\subsection{Characterization of self-assembled PMMA photonic crystal}

Both the structural and optical characterization of the PMMA photonic crystal are carried out to observe the lattice arrangement and the bandgap property of the crystal. The structural characterization is done by imaging the crystal by SEM, $\mathrm{OM}$ and atomic force microscope (AFM). Figure 4 is the SEM image of the plane of the crystal which is parallel to the substrate. The hexagonal arrangement of the spheres in this plane represents the $\left(\begin{array}{lll}1 & 1 & 1\end{array}\right)$ plane of face centred cubic lattice arrangement. Many crystalline domains separated by grain boundaries are seen in the optical microscope image (not shown here). However, all the domains reflect the same colour representing single crystalline arrangement of the spheres.

The AFM image is taken in the tapping mode. It is a nondestructive process to study the surface quality of the crystal. Figure 5 is the AFM image of the crystal. The roughness of the surface of the fabricated crystal for $5 \times 5 \mu \mathrm{m}^{2}$ area is $17.5 \mathrm{~nm}$ which represents a very uniform arrangement of spheres. In the AFM technique, the charging effect and the damage of the sample due to high voltage can be avoided unlike in the case of SEM technique. It can also be used to get an average diameter of the colloids in the scanned area.

Measurement of the reflection spectrum provides the necessary optical characterization. The stop band of the given crystal is measured from the reflection spectrum taken at different angles of incidence. Perkin-Elmer Lambda 950 spectrophotometer with a universal reflectance accessory is used to measure the spectrum using a Halogen lamp as the source.

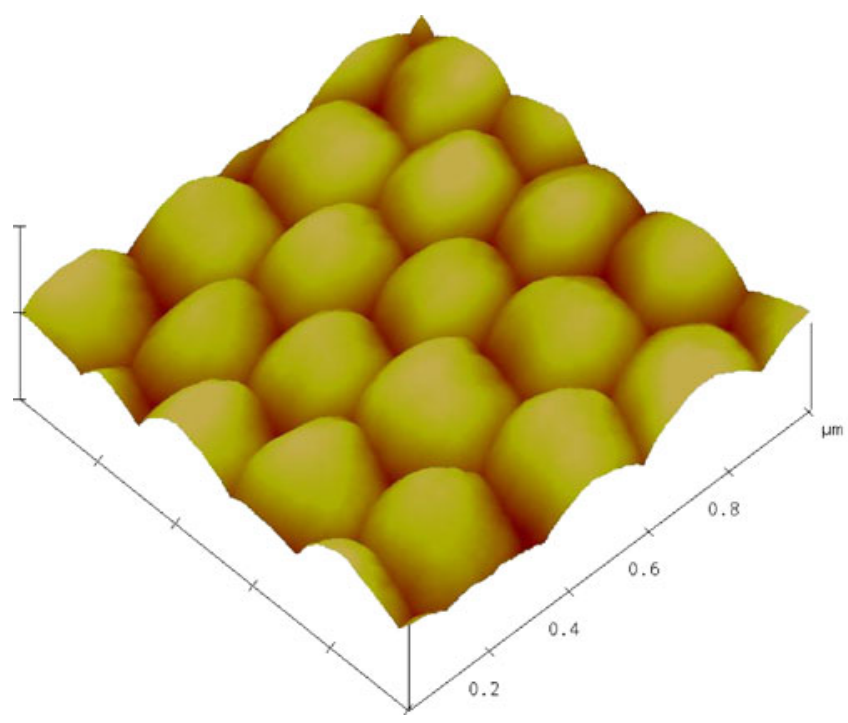

Figure 5. AFM image of PMMA photonic crystal showing plane parallel to substrate $\left(\right.$ scan area $\left.1 \times 1 \mu^{2}\right)$.

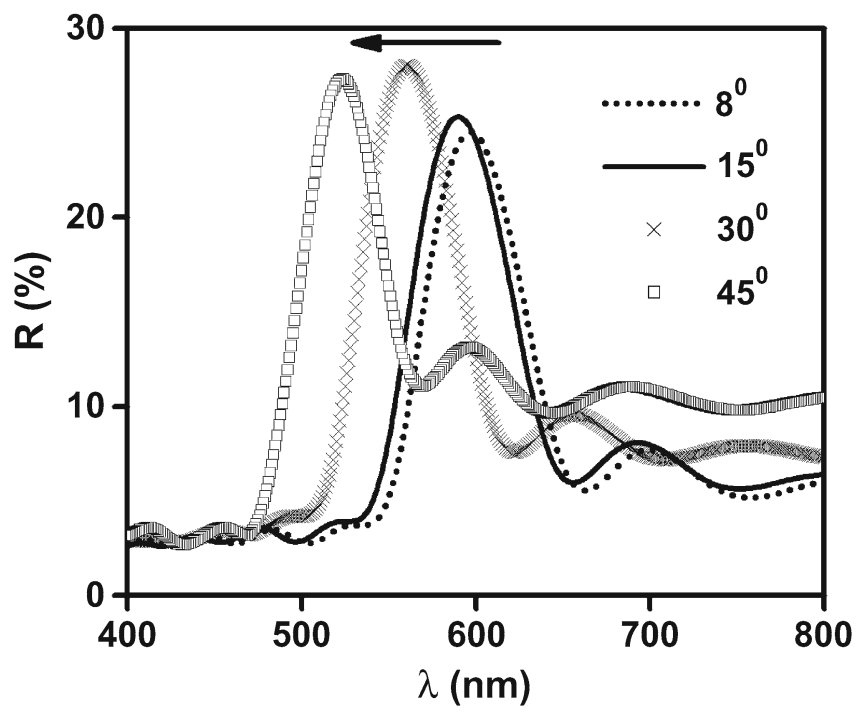

Figure 6. Reflection spectrum of 3D photonic crystal fabricated from a PMMA colloidal solution of sphere diameter $287 \mathrm{~nm}$ at different angle of incidence. The arrow indicates the direction of increasing angle.

The spot size of the beam on the sample is $12.5 \times 5 \mathrm{~mm}^{2}$ which will cover multiple single-crystalline domains on the crystal surface. The incident light is unpolarized.

Figure 6 is the reflection spectrum of PMMA photonic crystal for different angles of incidence of light. Under nearnormal incidence $\left(8^{\circ}\right)$, the crystal shows a peak reflectance of $25 \%$ at $597 \mathrm{~nm}$. The value of peak reflectance is governed by the number of well-ordered layers and the index contrast between the spheres and the voids. The wavelength of peak 


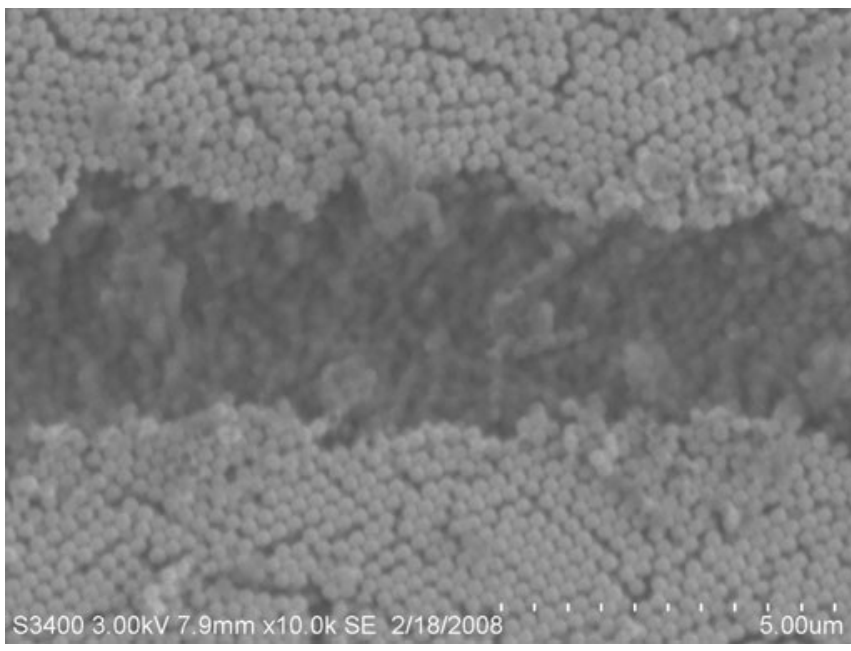

Figure 7. SEM image of a channel waveguide on 3D ordered photonic crystal (scale bar $5 \mu \mathrm{m}$ ).

reflection is decided by the periodicity of the sample and the index contrast. The diameter $D$ of the colloids is $287 \pm$ $8 \mathrm{~nm}$. This nearly mono-disperse condition is required for fabricating well-ordered photonic crystals. The lattice period in the $\left(\begin{array}{lll}1 & 1 & 1\end{array}\right)$ direction is $d_{111}=0.816 D=234 \mathrm{~nm}$. The effective index of the sample is 1.37. Assuming an ideal fill fraction of $74 \%$ in the face centred cubic arrangement, the wavelength of peak reflectance is expected at $645 \mathrm{~nm}$ for normal incidence. The experimentally observed value is lower due to the non-ideal crystal structure resulting from the selfassembly process. The reflection peak shifts from $597 \mathrm{~nm}$ to $521 \mathrm{~nm}$ as the angle of incidence increases from $8^{\circ}$ to $45^{\circ}$. With the increase in angle of incidence, the peak of the spectrum moves towards the lower wavelength region, as expected from the modified Bragg's law, and the peak reflectance value remains nearly constant. As angle of incidence increases, the beam sees a larger area of the crystal. More defects and disorder present in the larger area should decrease the peak value of reflection. In the present work, the peak reflectance is nearly constant for different angles of incidence, indicating the good quality of the crystal arrangement. The given crystal is a pseudo-bandgap material due to the low refractive index contrast between the solid PMMA spheres and the air voids (refractive index contrast is 0.49 ).

\subsection{Characterization of waveguides fabricated on photonic crystal}

Figure 7 is the SEM image of single channel waveguide fabricated on the photonic crystal. In this figure, the dark portion is the air channel which is surrounded by ordered spheres. A very high resolution and magnification in SEM is required to show the arrangement of spheres at the depth of the air channel, since the waveguide depth is less than the overall

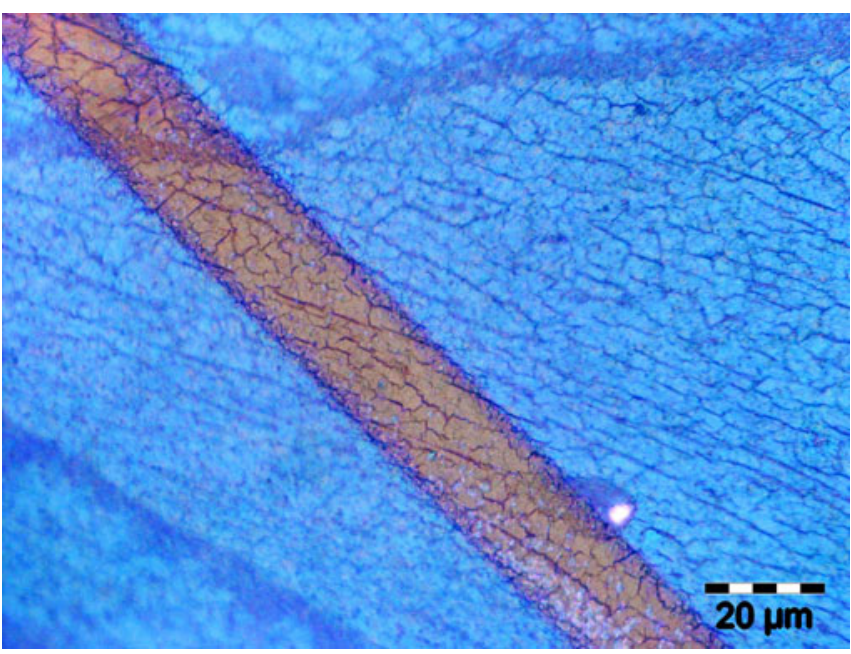

Figure 8. Optical microscope image of channel waveguide on PMMA photonic crystal (scale bar $20 \mu \mathrm{m}$ ).

crystal thickness. Figure 8 is the OM image, in which the red colour band is the air channel. The difference in colour of air channel compared to the colour of the photonic crystal (blue) represents the change in surface quality of the crystal in the channel region. The lines in the red band are the grain boundaries in the photonic crystal present at the base of the waveguide.

When the wavelength of light launched into the channel waveguide is comparable to the bandgap of the photonic crystal, it will undergo high reflection at the interface due to Bragg reflection from the crystal planes and propagate inside the channel by multiple reflections. It is important to notice that different modes may reflect to different extents at the air-photonic crystal interface due to the pseudo-band gap effect. Light can travel in the rarer medium surrounded by the denser medium (photonic crystal) on the three sides, only in the presence of a complete bandgap. This requires an increase of the index contrast which can be achieved by infiltration of the photonic crystal with high-index materials in its unexposed region. In addition, a complete optical characterization of this waveguide structure for the allowed guided modes requires a tunable laser.

It is found that a relatively lesser accelerating voltage is required to write waveguide structures on PMMA photonic crystal as compared to the PMMA thin film. This is because the molecular weight of PMMA polymer used to fabricate the thin film is higher as compared to the molecular weight of PMMA colloidal spheres. In addition, since the photonic crystal is an arrangement of sub-micron particles, the property of PMMA in the photonic crystal is different from that in the bulk (thin film). In spite of these differences, PMMA can be used effectively to design waveguide structures in the bulk form of electron-beam resist as well as in the microstructured self-assembled photonic crystals. Accelerating voltage and beam current for EBL writing require an 
optimization depending upon the molecular weight and the microstructure of the resist. Further optical characterization of the waveguide structure is in progress.

\section{Conclusions}

The details of fabrication of waveguide structures on PMMA thin film by EBL method are presented. The structural characterization of the fabricated structures is obtained from SEM and OM images. Inward-growing self-assembly method is used for fabrication of three-dimensionally ordered PMMA photonic crystals. Well-ordered photonic crystals were obtained by this technique. The structural and optical characterizations of the fabricated photonic crystals show a face centred cubic lattice arrangement and a pseudo-bandgap near $600 \mathrm{~nm}$, respectively. Channel waveguide structure is written on the photonic crystal by EBL technique under optimized conditions of accelerating voltage and beam current. The structural characterization of the fabricated channel waveguide indicates the success of this method.

The waveguide structures fabricated in this work can be used for the guidance of light (a) in the denser medium for the thin film case and (b) in a rarer medium (air) with photonic crystal structure as a cladding. The light launched in photonic crystal waveguides will face an effective refractive index in the cladding, similar to the case in photonic crystal fibres (Knight 2003). The guided modes in the photonic crystal waveguide structure can be tuned by controlling the lattice constant and the effective refractive index of the surrounding crystal. The effective index of the crystal can be modified by infiltrating the air voids of the crystal with higher index materials such as ethanol, ethylene glycol, silica and zinc oxide, thus paving the way for photonic crystal-based sensor applications (Nair and Vijaya 2010).

\section{Acknowledgements}

One of the authors (SK) is financially supported by the Board of Research in Nuclear Sciences (BRNS), Government of India. This work was sponsored by the Principal Scientific Advisor to the Government of India. The authors gratefully acknowledge the technical assistance at the EBL fabrication laboratory, Electrical Engineering Department, IIT Bombay.

\section{References}

Ferrand P, Egen M, Zentel R, Seekamp J, Romanov S G and Torres C M S 2003 Appl. Phys. Lett. 255289

Ferrand P, Seekamp J, Egen M, Zentel R, Romanov S G and Torres C M S 2004 Microelectron. Eng. 73-74 362

Fu C B, Yang C S, Kuo M C, Lai Y J, Lee J, Shen J L, Chou W C and Jeng S 2003 Chinese J. Phys. 5535

Joannopoulos J D, Meade R D and Winn J N 1995 Photonic crystals: Molding the flow of light (Princeton, NJ: Princeton University Press)

John S 1987 Phys. Rev. Lett. 232486

John S and Quang T 1995 Phys. Rev. Lett. 173419

Knight J C 2003 Nature 424847

Mukherjee N, Eapen B J, Keicher D M, Luong S Q and Mukherjee A 1995 Appl. Phys. Lett. 253715

Nair R V and Vijaya R 2008 Appl. Phys. A90 559

Nair R V and Vijaya R 2010 Prog. Quant. Electr. 3489

Panepucci R R, Kim B H, Almeida V R and Jones M D 2004 J. Vac. Sci. Technol. B22 3348

Stodolka J, Nau D, Frommberger M, Zanke C, Giessen H and Quandt E 2005 Microelectron. Eng. 78-79 442

Tseng A A, Chen K, Chen C D and Ma K J 2003 IEEE Transactions on electronics packaging manufacturing 2141

Yablonovitch E 1987 Phys. Rev. Lett. 202059

Yablonovitch E 1993 J. Opt. Soc. Am. B2 283

Yan Q, Zhou Z and Zhao X S 2005 Langmuir 73158 